\title{
Blind Channel Equalization by Adaptive Filter Algorithms
}

\author{
S. Elkassimi ${ }^{1, *}$, S. Safi ${ }^{1}$, B. Manaut ${ }^{2}$ \\ ${ }^{1}$ Department of Mathematics and Informatics, Polydisciplinary Faculty, Sultan Moulay Slimane University, Morocco \\ ${ }^{2}$ Department of Physics, Polydisciplinary Faculty, Sultan Moulay Slimane University, Morocco
}

Copyright $(2017$ by authors, all rights reserved. Authors agree that this article remains permanently open access under the terms of the Creative Commons Attribution License 4.0 International License

\begin{abstract}
This paper propose an algorithm based on $\mathrm{ZF}$ and MMSE methods for blind channel equalization, which is compared with adaptive filter algorithms which are Constant Modulus Algorithm (CMA), Fractional Space CMA (FSCMA) and Sign Kurtosis Maximization Adaptive Algorithm (SKMAA). The simulations show that the proposed algorithm gives satisfied result versus CMA, FSCMA and SKMAA algorithms. The study is done under certain conditions, it is implemented in noisy environment, for different number of symbols and different SNR values with QPSK modulation. Equalization of channel is more performing if we use the proposed algorithms.
\end{abstract}

Keywords Blind Equalization, CMA, FSCMA, SKMAA, ZF, MMSE, SER

\section{Introduction}

Conventional equalization and carrier recovery algorithms for Minimizing Mean Square Error (MMSE) in digital communication systems generally require an initial training period during which a known data sequence is transmitted and properly synchronized at the receiver $[1,2]$. On the other side the blind channel equalization allows to retrieve the unknown input sequence symbol to the remote channel without the use of training bits. In addition blind channel equalization has become an important topic in digital communications [3]. Blind methods use the received signal sequence and some a priori knowledge of the input sequence statistics [4]. Non-minimum phase channel equalization was performed using the high-order statistics methods [5-8] or other nonlinearities that are effective only with non-Gaussian distribution input sequences [9]. In this paper, we study the most popular adaptive blind equalization Bussgang algorithm (the Godard algorithm [10]) or constant modulus (CMA) [11] and Fraction Spaced CMA algorithms [10,12], thus another algorithm based on Kurtosis Maximization method called Sign Kurtosis Maximization Adaptive algorithm (SKMAA) [13]. In telecommunication, equalization means filtering the noise which is in a signal transmitted through a channel. Conventional channel equalization uses an initial training phase, while blind channel equalization does not use this phase. According to the study, there are some well know algorithm for blind channel equalization, these algorithms, like CMA, FSCMA and SKMAA, are based on adaptive filter techniques. In this paper we propose a new algorithm, which is based on ZF and MMSE methods. The result of this algorithm looks better comparatively to the CMA, FS-CMA and SKMAA algorithm.

\section{Problem Statement}

The symbols of blind equalization by adaptive filter algorithms are depicted in Fig. 1. Let us assume that the transmitted symbol sequences, $S(k)$, are independent and identically distributed (i.i.d), for $S(k) \in A$. Where $A$ is the windows character set.

The input equalizer is defined by:

$$
\begin{aligned}
& X(k)=h(k) \cdot S(k)+V(k) \\
& =\sum_{i=0}^{N} h(i) \cdot S(k-i)+V(k)
\end{aligned}
$$

Where $h=[h(1), h(2), \ldots, h(k)]$ represent the channel parameters, $S(k)$ is the input system and $V(k)$ is the additive colored Gaussian noise.

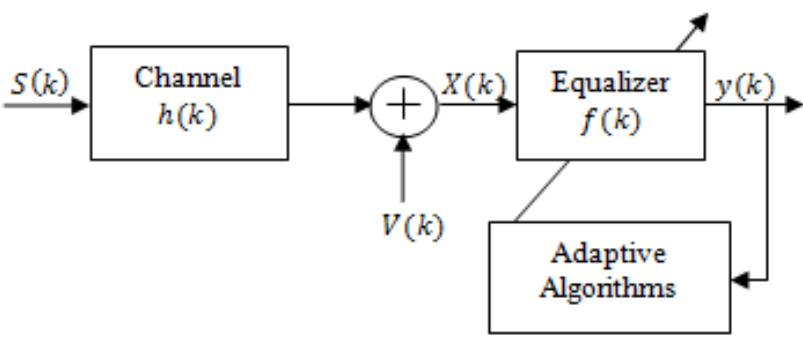

Figure 1. Blind Equalization system model 
The output of the equalizer can be defined by the following relationship

$$
y(k)=f(k) \cdot X(k)
$$

Where $f(k)$ is the tap weight vector and $X(k)$ is the input data vector of the equalizer at $\mathrm{k}^{\text {th }}$ instant sampling time.

At sample $\mathrm{k}$, the output of the equalizer is expressed by the relationship

$$
y(k)=\sum_{i=0}^{N} f(i) \cdot X(k-i)
$$

In this paper, we study the blind channel equalization using the adaptive filter algorithms. In addition we represent a comparison between the proposed algorithm and the adaptive filter algorithms such as the Constant Modulus Algorithm (CMA), Fractional-Spaced-CMA, and Sign Kurtosis Maximization Adaptive Algorithm (SKMAA).

\section{Blind Channel Equalization}

The aim of blind channel equalization is to retrieve the unknown input sequences symbol to the remote channel. That is to say: calculate the Symbol Error Rate (SER). In this case we propose an algorithm based on ZF and MMSE equalizer, which is compared to the adaptive filter algorithms.

In the first step we describe the proposed algorithm with ZF and MMSE methods, and the second step we describe the adaptive filter algorithms such as CMA, FSCMA and SKMAA.

\section{A. Proposed Algorithm (Said1)}

The proposed algorithm (Said1) is based on ZF and MMSE equalizer, this algorithm allow to blind channel equalization,

\section{With ZF Equalization}

In order to compensate the effect of power line communication (PLC) channel, we consider the ZF equalizer, which satisfies the condition shown below (Eq. 4).

$$
f_{Z F} H=I
$$

Where $f_{Z F}=\left(H^{H} H\right)^{-1} H^{H}$ is the ZF decoding matrix, $(.)^{H}$ denotes Hermitian transpose, $H$ is the channel matrix and $I$ is the identity matrix. Given the received signal $X$, the receiver can obtain the estimated signal by using the ZF equalization, which is given by:

$$
\begin{gathered}
\hat{S}(n)=f_{Z F} X(n) \\
\hat{S}(n)=f_{Z F} H S(n)+f_{Z F} V(n) \\
\hat{S}(n)=I S(n)+f_{Z F} V(n)
\end{gathered}
$$

Where $\hat{S}$ is the estimated matrix of the transmitted signal. If the determinant of $H$ is not zero, so, the inverse matrix of $H$ exists and the decoding matrix can be expressed as

$$
f_{Z F}=H^{-1}
$$

The ZF equalization is ideal when the channel is not affected by the noise. However, when the channel is noisy, the ZF equalization will amplify the noise greatly where the channel has small magnitude in the attempt to invert the channel completely.

\section{With MMSE Equalization}

In order to minimize the power of the noise component, we employ the MMSE equalization, which is given by:

$$
f_{M M S E}=\underbrace{\min }_{f_{M M S E} \min } E\left|\left\|S_{n-d}-f_{M M S E}^{H} X(n)\right\|^{2}\right|
$$

Where $f_{M M S E}$ is the MMSE decoding matrix and $\|$. represent the norm of the value $\left(S_{n-d}-f_{M M S E}^{H} X(n)\right.$.)

Or:

$$
f_{M M S E}=R_{X} \hat{h}_{d}
$$

Where $R_{X}=E\left[X(n) X^{H}(n)\right]$ and $\hat{h}_{d}=E\left[X(n) S_{n-d}^{*}\right]$. In equation 9 and 10 we represent the expression of $R_{X}$ and $\widehat{h}_{d}$

$$
\begin{aligned}
R_{X} & =\frac{1}{k} \sum_{n=1}^{k} X(n) X^{H}(n) \\
\hat{h}_{d} & =\frac{1}{M} \sum_{n=1}^{M} X(n) S_{n-d}^{*}
\end{aligned}
$$

The MMSE equalization can be used to estimate the channel effect with varying the decoding matrix in accordance with SNR. Besides, it prevents the noise component from being amplified.

The steps of the proposed algorithm are summarized in the following:

- Transmit $\mathrm{k}$ symbols, the first M symbols are assumed known,

- Obtain received samples $X_{n}$,

- Construct sample vectors $X(n)$,

- Calculate $R_{X}$ and $\hat{h}_{d}$,

- Calculate $f_{M M S E}=R_{X}^{-1} \hat{h}_{d}$,

- Calculate the symbol error rate (SER):

- Use $f^{H} X(n)$ to estimate symbol $\hat{S}_{n-d}$.

- $\quad$ Compare $\hat{S}_{n-d}$ with $S_{n-d}$ value.

\section{B. CMA Equalizer[14-16]}

The problem with blind adaptation techniques is their poor convergence property compared to traditional techniques using training sequences. Generally a gradient descent based algorithm is used with the blind adaptation schemes. The most commonly used gradient descent based blind adaptation algorithm is the Constant Modulus Algorithm (CMA). The Constant Modulus Algorithm (CMA) has gained widespread practical use as a blind adaptive equalization algorithm for digital communications systems operating over inter-symbol interference channels.

The constant modulus (CM) criterion can be expressed by the cost function $J_{C M}=\frac{1}{4} E\left\{\left(\left|X_{n}\right|^{2}-\gamma\right)^{2}\right\}$, where $\gamma$ is a positive constant known as the Godard radius. The equalizer update algorithm leading to a stochastic gradient descent of 
$J_{C M}$ is known as the Constant Modulus Algorithm (CMA) and is specified by [9]:

$$
f(n+1)=f(n)+\mu S^{*}(n) \underbrace{X_{n}(\gamma(X(n))}_{\triangleq}
$$

Where $\mu$ is a step-size and $S^{*}(n)$ is the equalizer input vector at time index $\mathrm{n}$. the asterisk denotes conjugation. The function $\psi($.$) identified in (11) is referred to as the CMA$ error function.

\section{Fractional Spaced CMA Equalizer}

For a Fractionally Spaced Equalizer (FSE) [17], the tap spacing of the equalizer is a fraction of the baud spacing (in time) or the transmitted symbol period. As the output of the equalizer has the same rate as the input symbol rate, the output of the FSE needs to be calculated once in every symbol period [11]. Fractional Spaced CMA (FSCMA) algorithm uses directly the estimated coefficients of the equalizer. In fractional space the global convergences is fixed by [18]:

$$
\min J=E\left[\left(\left|f^{H} X(n)\right|^{2}-R_{z}\right)^{2}\right]
$$

Update rule:

$$
f_{n+1}=f_{n}-\mu \cdot E\left(\left|f^{H} X(n)\right|^{2}-R_{z}\right) X(n) X^{H}(n) f_{n}
$$

Algorithm:

- Construct the received sample space.

- Construct the sample vector $X(n)$.

- For $\mathrm{n}=1,2, \ldots$

- Update function (13)

- Calculate instantaneous error

- Check SER.

\section{SKMAA Equalizer[9]}

Sign Kurtosis Maximization Adaptive Algorithm (SKMAA) is used to blind channel equalization. The SKMAA is developed based on kurtosis of stochastic signals, such as: the stochastic ascend approach, and sign algorithm for restoring the blind equalizer weight vectors [19]. Furthermore it is also necessary to calculate the output sample $y(n)$ of the equalizer filter once per algorithm update. The general idea is to maximize the chosen cost function. To assure that the SKMAA algorithm ascends, for each time step $n$ the following requirement is made

$$
J(f(n+1))>J(f(n))
$$

Or the cost function is given by

$$
J_{K M A}(f)=\frac{|K u r t o s i s[y(n)]|}{E^{2}|y(n)|^{2}}
$$

where $y(n)$ is the equalizer output and $\operatorname{Kurtosis}[y(n)]$ is defined by:

$$
\operatorname{Kurtosis}[y(n)]=E\left\{y^{4}(n)\right\}-3 E^{2}\left\{y^{2}(n)\right\}
$$

The update is then given by

$$
\begin{gathered}
f(n+1)=f(n)+\mu \Delta f J_{K M A}(f) \\
=f(n)+\mu F(y)[S(n) * h(n)] \\
=f(n)+\mu F(y) X(n)
\end{gathered}
$$

or

$$
\Delta f J_{K M A}(f)=F(y) X(n)
$$

where $F$ is the feedback function defined by:

$$
F(y)=\frac{4\left(E\left[y^{2}\right] y^{2}-E\left[y^{4}\right]\right) y}{E^{3}\left[y^{2}\right]}
$$

a sign algorithm is introduced into KMAA. So the SKMAA is written by:

$$
f(n+1)=f(n)+\alpha \operatorname{Sign}[F(y)] \cdot[X(n)]
$$

where Sign[.] is a simple sign function, and $\alpha$ is a forgetting factor which is used to replace the ensemble

\begin{tabular}{|c|c|c|}
\hline SNR & Equalizer & SER \\
\hline $0 \mathrm{~dB}$ & $\begin{array}{c}\text { CMA } \\
\text { FSCMA } \\
\text { SKMAA }\end{array}$ & $\begin{array}{l}0.4628 \\
0.4373 \\
0.3127\end{array}$ \\
\hline $5 \mathrm{~dB}$ & $\begin{array}{c}\text { CMA } \\
\text { FSCMA } \\
\text { SKMAA }\end{array}$ & $\begin{array}{l}0.2789 \\
0.2554 \\
0.2278 \\
\end{array}$ \\
\hline $10 \mathrm{~dB}$ & $\begin{array}{c}\text { CMA } \\
\text { FSCMA } \\
\text { SKMAA }\end{array}$ & $\begin{array}{l}0.1881 \\
0.1793 \\
0.2061 \\
\end{array}$ \\
\hline $15 \mathrm{~dB}$ & $\begin{array}{c}\text { CMA } \\
\text { FSCMA } \\
\text { SKMAA }\end{array}$ & $\begin{array}{l}0.0369 \\
0.0337 \\
0.1745 \\
\end{array}$ \\
\hline $20 \mathrm{~dB}$ & $\begin{array}{c}\text { CMA } \\
\text { FSCMA } \\
\text { SKMAA }\end{array}$ & $\begin{array}{c}0.0070 \\
0 \\
0.1544 \\
\end{array}$ \\
\hline $25 \mathrm{~dB}$ & $\begin{array}{c}\text { CMA } \\
\text { FSCMA } \\
\text { SKMAA }\end{array}$ & $\begin{array}{c}0.0040 \\
0 \\
0.1474\end{array}$ \\
\hline
\end{tabular}
averages by empirical averages which are then adaptively updated $(0<\alpha<1)$.

\section{Simulation Results}

\section{A Performance of the Adaptive Filter Equalizer CMA, FSCMA and SKMAA}

In this part, we compare between the CMA, FSCMA and SKMAA equalizer that are being studied, for testing its performance. Tables 1 and 2 represent the Symbol Error Rate (SER) for different SNR values and number of symbols M.

Table 1. Comparison the Symbol Error Rate (SER) of different adaptive filter equalizer for complex channel with different SNR values 
Table 2. Comparison the Symbol Error Rate (SER) of different adaptive filter equalizer for complex channel with different number of symbols

\begin{tabular}{|c|c|c|}
\hline Number of Symbols M & Equalizer & SER \\
\hline \multirow{3}{*}{512} & CMA & 0.4076 \\
& FSCMA & 0.3659 \\
& SKMAA & 0.3150 \\
\hline \multirow{2}{*}{1024} & CMA & 0.4772 \\
& FSCMA & 0.3944 \\
& SKMAA & 0.3197 \\
\hline \multirow{2}{*}{2048} & CMA & 0.5059 \\
& FSCMA & 0.4792 \\
& SKMAA & 0.3481 \\
\hline \multirow{2}{*}{4096} & CMA & 0.5563 \\
& FSCMA & 0.5177 \\
& SKMAA & 0.3658 \\
\hline
\end{tabular}

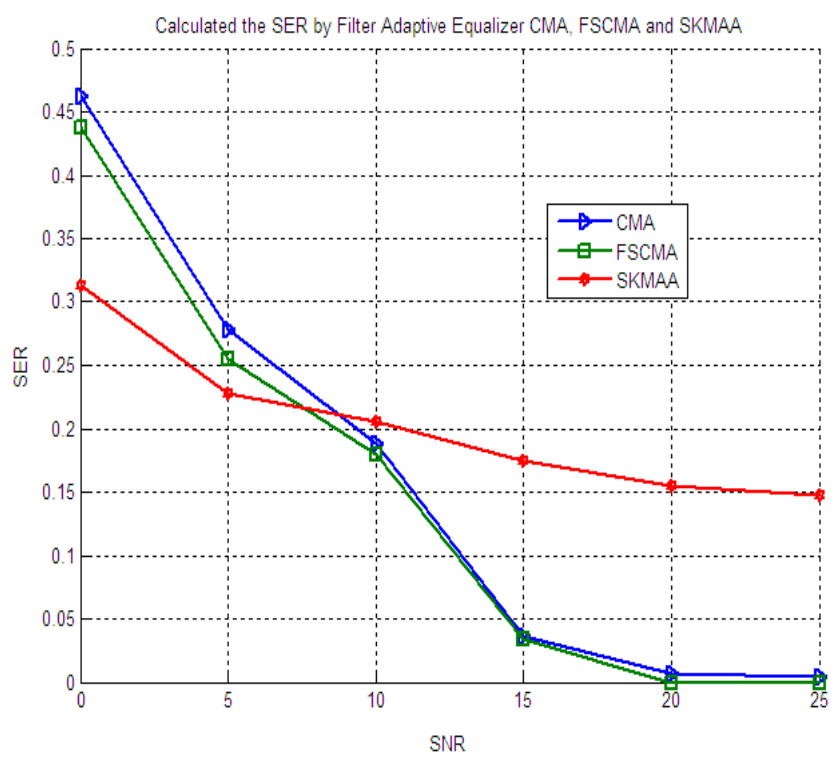

Figure 2. SER in function of SNR Values using CMA, FSCMA and SKMAA Equalizer

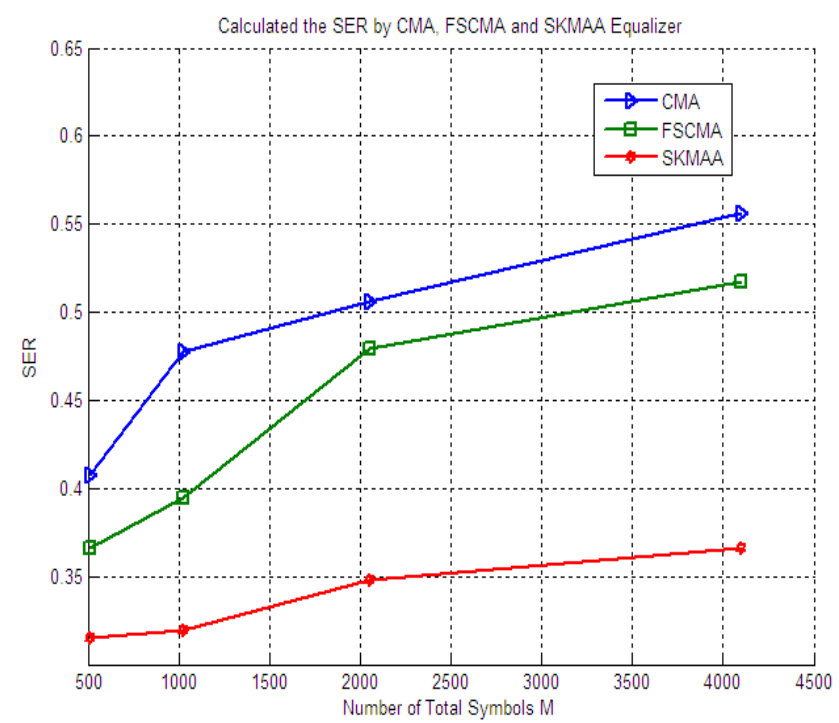

Figure 3. SER in function of number of symbols $M$ using CMA, FSCMA and SKMAA Equalizer.

From the figure 2, we remark that the SKMAA equalizer is more performing in noisy environment $(\mathrm{SNR}<10 \mathrm{~dB})$ than CMA and FSCMA equalizer. But if SNR $>10 \mathrm{~dB}$ we remark that CMA and FSCMA algorithms are more performing than SKMAA algorithm. In addition SKMAA equalizer is better than CMA and FSCMA equalizer, if the number of symbols $\mathrm{M}$ is larger (Fig. 3).

\section{B Performance of the proposed algorithm versus Adaptive Filter Equalizer}

In this part we test the performance of proposed algorithm (Said1), and adaptive filter equalizer (CMA, FSCMA and SKMAA) for blind channel equalizer in the following conditions:

The impulse response of complex channel is given by

$\mathrm{h}=\left[\begin{array}{lll}0.0545+\mathrm{j} * 0.05 & 0.2832-.1197 * \mathrm{j} & 0.7676+0.2788 * \mathrm{j}\end{array}\right.$ 0.0641-.0576*j 0 .0566-.2275*j $0.4063-0.0739 * \mathrm{j}]$, with different SNR values, the total number of data is $\mathrm{T}=1048$ and we use the QPSK or 4 QAM modulation. 

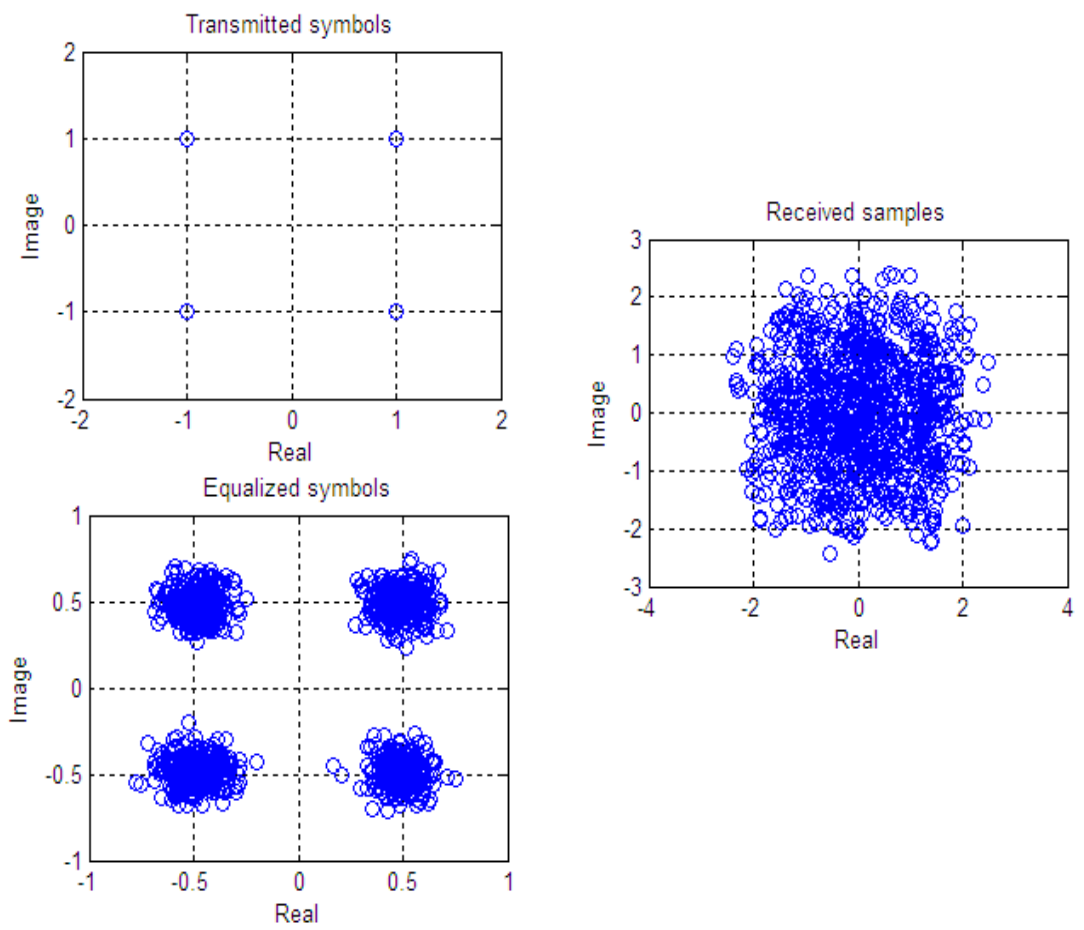

Figure 4. Channel equalization by proposed algorithm (Said1) with $\mathrm{SNR}=25 \mathrm{~dB}$.
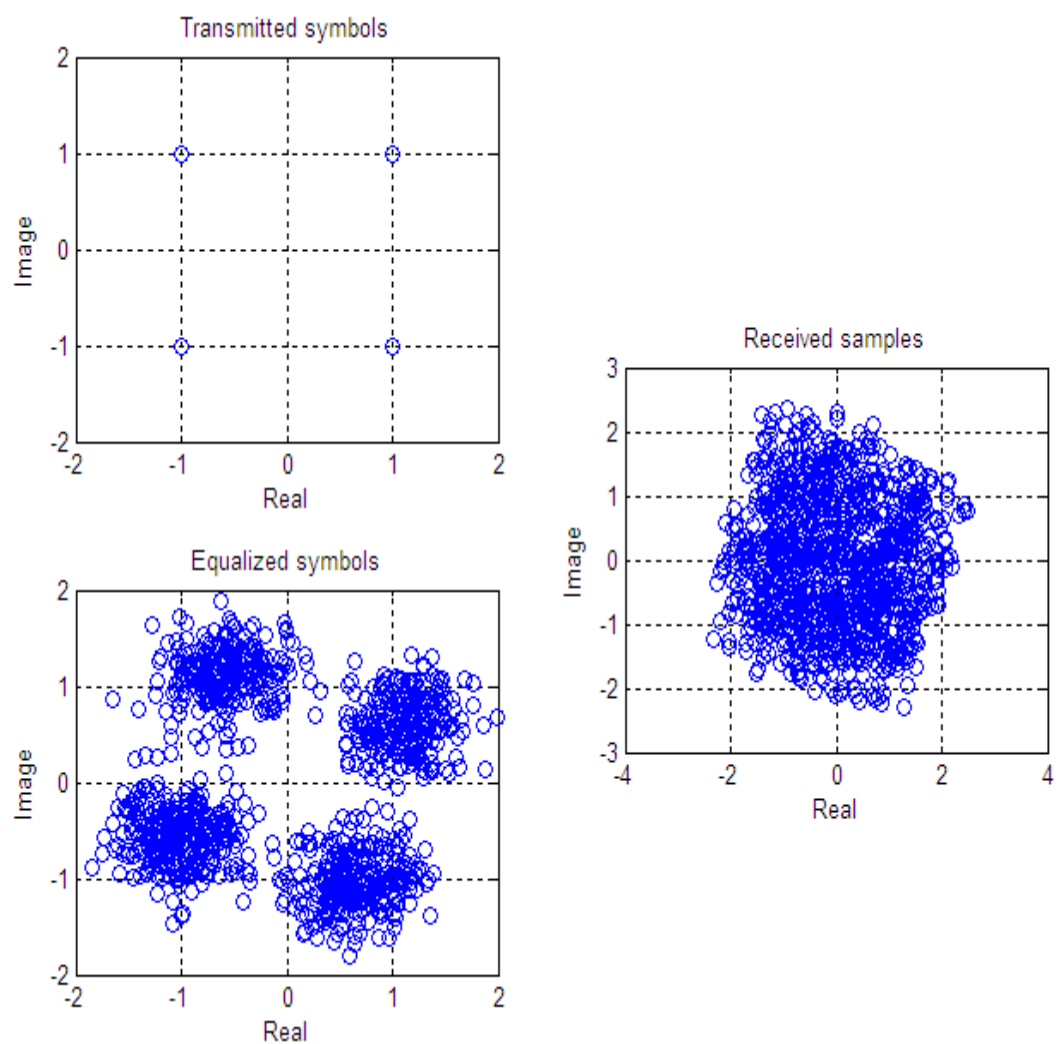

Figure 5. Channel equalization by $\mathrm{CMA}$ equalizer with $\mathrm{SNR}=25 \mathrm{~dB}$. 

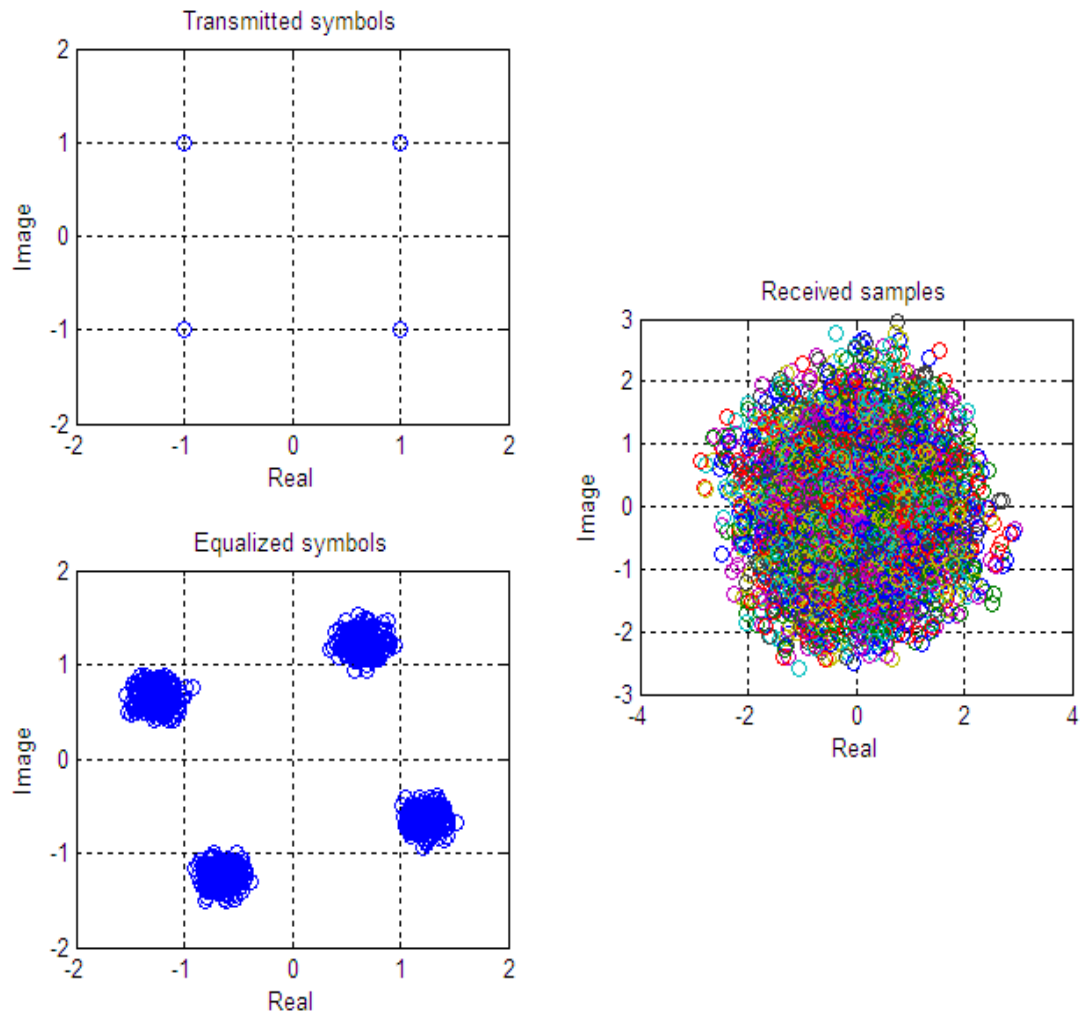

Figure 6. Channel equalization by FSCMA equalizer with $\mathrm{SNR}=25 \mathrm{~dB}$.
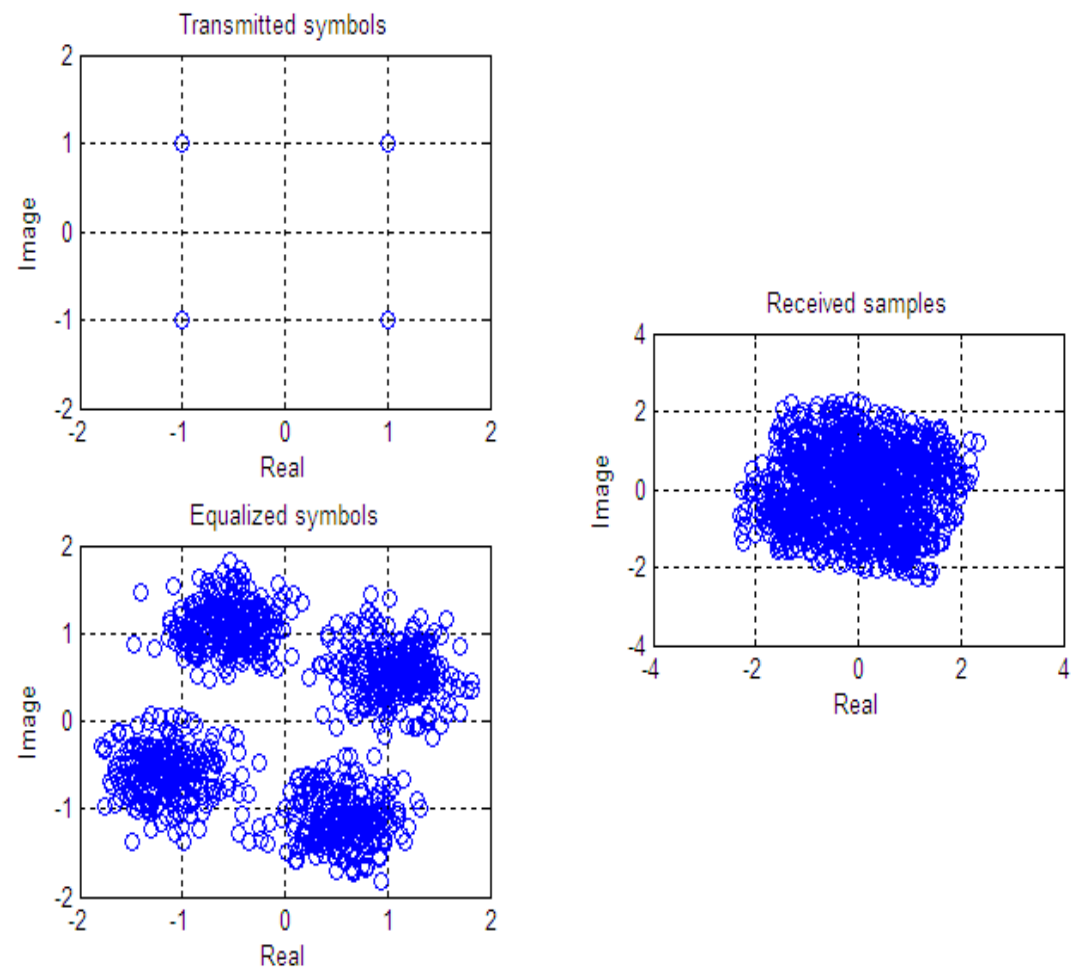

Figure 7. Channel equalization by SKMAA equalizer with $\mathrm{SNR}=25 \mathrm{~dB}$. 


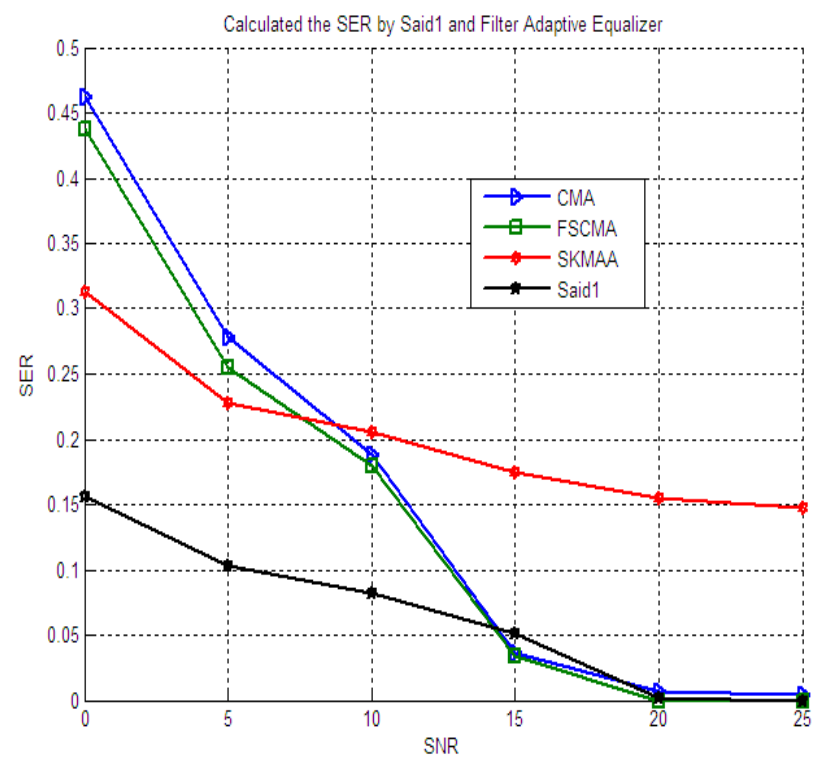

Figure 8. SER in function of SNR values using proposed algorithm (Said1), CMA, FSCMA and SKMAA equalizer

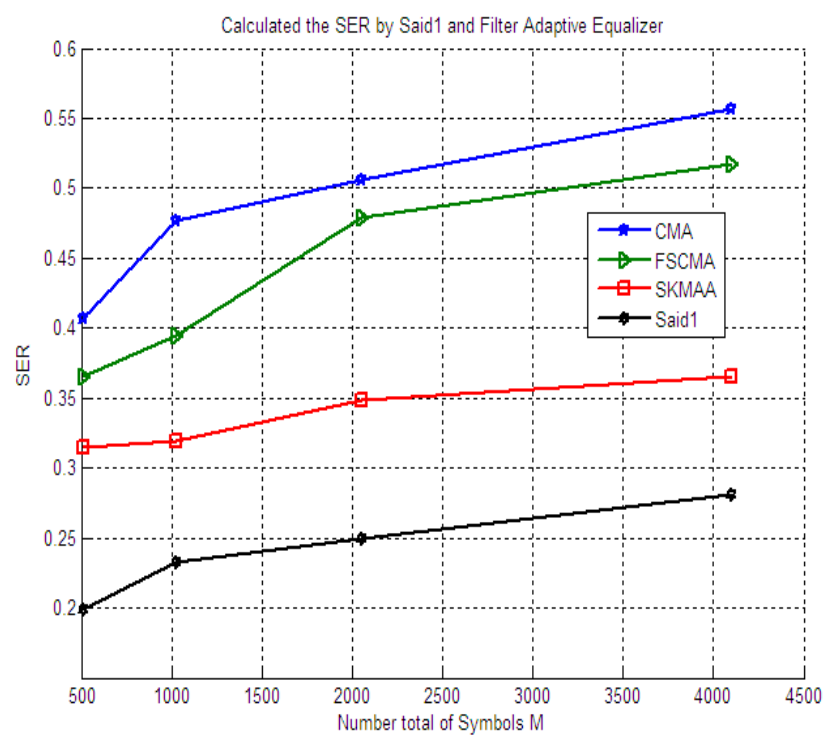

Figure 9. SER in function the number total of symbols $M$ using proposed algorithm (Said1), CMA, FSCMA and SKMAA equalizer.

From the figure 8 and 9 we note that the proposed algorithm (Said1) gives a good channel equalization compared to CMA, FSCMA and SKMAA equalizer. In addition the SER is decrease according to SNR values and increase according to total symbols $\mathrm{M}$.

\section{Conclusions}

In this paper we have proposed an algorithm based on ZF and MMSE methods for blind channel equalization. This algorithm was compared with three filter adaptive algorithms such as: CMA, FSCMA and SKMAA. The simulation results demonstrates that the proposed algorithm gives an efficient equalization of channel, that is to say, gives a good SER values versus to CMA, FSCMA and SKMAA equalizer.

\section{REFERENCES}

[1] Godard, D., "Self-Recovering Equalization and Carrier Tracking in Two-Dimensional Data Communication Systems", Communications, IEEE Transact, v.28, Issue.11, pp 1867-1875, Nov 1980.

[2] John G. Proakis, "Digital Communications", McGraw Hill Higher Education, 4e édition, Paperback (December 1, 2000).

[3] L. R. Litwin, Jr., "Blind channel equalization", IEEE Potentials, vol. 18, no. 4, pp. 9-12, Apr. 1999.

[4] Elisabeth de Carvalho, Member, IEEE, Dirk Slock, Member, IEEE, "Blind and Semi-Blind FIR Multichannel Estimation: (Global) Identifiability Conditions", IEEE Trans. Signal Process, Vol. 52, 2004.

[5] O. Shalvi and E. Weinstein, "New criteria for blind deconvolution of non-minimum phase systems (channels)", Information Theory, IEEE Transactions on, vol. 36, pp. 312-321, March 1990.

[6] B. Porat and B. Friedlander, "Blind equalization of digital communication channels using higher-order moments", IEEE Trans. Signal Processing, vol. 39, pp. 522-526, Feb. 1991.

[7] L. Tong, G. Xu and T. Kailath, "Blind identification and equalization based on second-order statistics: A time domain approach", IEEE Trans. Inform. Theory, vol. 40, pp. 340-349, Mar. 1994.

[8] M. Boulouird, M.M. Hassani, A. Zeroual, "Blind channel identification using higher-order statistics", Vol. 78, No.4, pp. 325-338, April 2008.

[9] I. Fijalkow, C.E. Manlove, and C. R. Johnson, Jr," Adaptive Fractionally Spaced Blind CMA Equalization: Excess MSE," IEEE Trans. Signal Processing, vol. 46, no. 1, january1998.

[10] Fijalkow, I., C. E. Manlove, and C. R. Johnson, Jr., "Adaptive fractionally spaced blind CMA equalization: Excess MSE," IEEE Trans. on Signal Processing, Vol. 46, No. 1, 227-231, 1998.

[11] J. R. Treichler and B. G. Agee, "A new approach to multipath correctionof constant modulus signals," IEEE Trans. Acoust., Speech, Signal Processing, vol. ASSP-31, pp. 459-472, Apr. 1983.

[12] I. Fijalkow, A. Touzni, and J. Treichler, "Fractionally spaced equalization using cma: Robustness to channel noise and lack of disparity." IEEE Trans. on Signal Proc., vol. 45, pp. 56-66, Jan. 1997.

[13] B. Siva Kumar Reddy, B. Lakshmi, "Minimizing PAPR and Synchronization Errors in OFDM for WiMAX Using Software Defined Radio", Journal of Circuits, Systems and Computers (World Scientific), Vol. 24, No. 4, pp: 1-23, 2015.

[14] D.N. Godard, "Self-recovering equalization and carrier tracking in two-dimensional data communication systems", IEEE Trans. on Communications, vol. 28, no. 11, pp. 1867-1875, Nov 1980. 
[15] J.R. Treichler and B.G. Agee, "A new approach to multipathcorrection of constant modulus signals," IEEE Trans Acoust. Speech Signal Proc., vol. ASSP-31, no.2, Apr. 1983.

[16] C. R. Johnson, P. Schniter and J. T. Endres, "Blind equalization using the constant modulus criterion: a review," Proc. of the IEEE, vol.86, no. 10, pp.1927-1949, Oct.1998.

[17] Gitlin, R.D. and Weinstein, S.B., "Fractionally spaced equalization: an improved digital transversal equalizer", Bell Syst. Tech. J., 60:275-296, 1981.

[18] Li, Y. and Ding, Z., "Global convergence of fractionally spaced Godard adaptive equalizers", IEEE Trans. Signal Process., SP-44:818-826, Apr., 1996.

[19] Xu, Zhengyuan and Liu, Ping, "Blind multiuser detection by kurtosis maximization/ minimization", IEEE Signal Processing Letters 55 pp.1-4, 2004.
[20] J.-N. Lin and R. Unbehauen, "Bias-remedy least mean square equation error algorithm for IIR parameter recursive estimation," IEEE Trans. Signal Processing, vol. 40, pp. 62-69, Jan. 1992.

[21] M. Boulouird, M.M. Hassani, A. Zeroual, "Blindchannel identification using higher-order statistics", Vol. 78, No.4, April 2008, 325-338.

[22] J.-T. Yuan and T.-C. Lin, "Equalization and carrier phase recovery of CMA and MMA in blind adaptive receivers", IEEE Trans. Signal Process. Vol.55, pp.3206-3217, 2010.

[23] A. J. Bell and S. T. J., "An information-maximization approach to blind separation and blind deconvolution", Neural Computation, vol. 7, pp. 1129-1159, 1995. 